OPEN ACCESS

Edited by: John Zaunders,

University of New South Wales, Australia

Reviewed by: John J. Miles,

Queensland Institute of Medical Research, Australia Karin Schilbach, University of Tübingen, Germany

*Correspondence: Daniela Weiskopf dweiskopf@lij.org

Specialty section: This article was submitted to T Cell Biology,

a section of the journal

Frontiers in Immunology

Received: 20 September 2016 Accepted: 10 November 2016 Published: 07 December 2016

Citation:

Tian Y, Sette A and Weiskopf D (2016) Cytotoxic CD4 T Cells:

Differentiation, Function, and Application to Dengue Virus Infection.

Front. Immunol. 7:531. doi: 10.3389/fimmu.2016.00531

\section{Cytotoxic CD4 T Cells: Differentiation, Function, and Application to Dengue Virus Infection}

\author{
Yuan Tian, Alessandro Sette and Daniela Weiskopf* \\ Division of Vaccine Discovery, La Jolla Institute for Allergy and Immunology, La Jolla, CA, USA
}

Dengue virus (DENV) has spread through most tropical and subtropical areas of the world and represents a serious public health problem. The control of DENV infection has not yet been fully successful due to lack of effective therapeutics or vaccines. Nevertheless, a better understanding of the immune responses against DENV infection may reveal new strategies for eliciting and improving antiviral immunity. $T$ cells provide protective immunity against various viral infections by generating effector cells that cooperate to eliminate antigens and memory cells that can survive for long periods with enhanced abilities to control recurring pathogens. Following activation, CD8 T cells can migrate to sites of infection and kill infected cells, whereas CD4 T cells contribute to the elimination of pathogens by trafficking to infected tissues and providing help to innate immune responses, B cells, as well as CD8 T cells. However, it is now evident that CD4 T cells can also perform cytotoxic functions and induce the apoptosis of target cells. Importantly, accumulating studies demonstrate that cytotoxic CD4 T cells develop following DENV infections and may play a crucial role in protecting the host from severe dengue disease. We review our current understanding of the differentiation and function of cytotoxic CD4 T cells, with a focus on DENV infection, and discuss the potential of harnessing these cells for the prevention and treatment of DENV infection and disease.

Keywords: CD4 T cells, cytotoxicity, dengue virus, protection, vaccines

\section{DENV INFECTION AND A PROTECTIVE ROLE FOR CYTOTOXIC CD4 T CELLS}

Dengue virus (DENV) is a major public health problem in tropical and subtropical areas with 390 million estimated infections per year (1). DENV has four serotypes (DENV 1-4), and infection with one of the serotypes can be asymptomatic or result in a range of diseases spanning from dengue fever (DF) to dengue hemorrhagic fever (DHF) and dengue shock syndrome (DSS). The more severe forms of DHF and DSS are more likely to develop following secondary infections with a different serotype (2). Other than supportive care there is currently no specific therapy available for the treatment of dengue diseases. Tremendous efforts have been devoted to the development of DENV vaccines since Word War II, and a tetravalent chimeric vaccine, Dengvaxia ${ }^{\circledR}$, has recently been licensed in several countries including Mexico, Brazil, and the Philippines (3). However, several clinical trials of Dengvaxia ${ }^{\circledR}$ raise concerns about the efficacy of the vaccine. A phase $2 \mathrm{~b}$ study in Thailand showed an overall efficacy of $30.2 \%$ with only $9.2 \%$ protection against DENV 2 (4). Additionally, two large-scale phase 3 trials in Asia and Latin America reported that the average efficacies against the four serotypes 
were 56.5 and $60.8 \%$, respectively, and further confirmed the lowest level of protection against DENV $2(5,6)$. Furthermore, a long-term follow up of these trials reveals that Dengvaxia ${ }^{\circledR}$ was less effective in seronegative vaccinees and resulted in an increased incidence of hospitalization among vaccinated children who were under 9 years old (7). Given the importance of host immunity in the protection of DENV infection, it is crucial to gain a better understanding of anti-DENV immune responses and identify the correlates of protection or susceptibility in order to improve the development of immunotherapies and vaccines for dengue disease.

$\mathrm{T}$ cells play important roles in fighting infections with intracellular pathogens; however, the roles of $\mathrm{T}$ cells during DENV infection may be complex. Although some studies suggest that $\mathrm{T}$ cells may contribute to the pathogenesis of DENV infection via the production of inflammatory cytokines, and that the expansion of preexisting cross-reactive memory $\mathrm{T}$ cells may impair viral control upon secondary heterologous infections (original antigenic sin), others indicate that $\mathrm{T}$ cells may play important roles in the protection against severe dengue disease (8). Stronger T cell responses generated following natural infection or vaccination with DENV as measured by the production of effector cytokines such as interferon- $\gamma($ IFN- $\gamma$ ) have been associated with better protection against subsequent DENV infection $(9,10)$. Additionally, our laboratory has demonstrated that protective human leukocyte antigen (HLA) alleles against dengue disease are associated with robust and polyfunctional CD8 T cell responses (11). Furthermore, the observation that the frequency of T cells that express CD107a, a degranulation marker, correlates with less severe dengue disease, supporting the notion that the roles of $\mathrm{T}$ cells during DENV infection may depend upon their functionality and that $\mathrm{T}$ cells with cytotoxic potentials may be crucial for the control of DENV infection (12).

Although cytotoxic functions are usually associated with CD8 $\mathrm{T}$ cells, accumulating evidence has demonstrated that a range of other cells can elicit cytotoxic effector functions. Dendritic cells (DCs) are the early, primary targets of DENV in natural infection, and the vigor of cell-mediated immunity is modulated by the relative presence or absence of IFN- $\gamma$ in the microenvironment surrounding the virus-infected DCs (13). DCs including Langerhans cells (LCs) express CD1d, a molecule responsible not only for the presentation of lipopeptides but also conventional antigens that have a specific binding motif, i.e., hydrophobic amino acids in position 1, 4, and 7 (14). CD1d-restricted natural killer T (NKT) cells are a distinct subset of $\mathrm{T}$ cells that rapidly produce an array of cytokines upon activation and play a critical role in regulating various immune responses. NKT cells are classified into two groups based on differences in T-cell receptor usage. Type I NKT cells have an invariant T-cell receptor $\alpha$-chain (iNKT), while Type II NKT cells have a more diverse T-cell receptor repertoire, and it has been shown that CD4 engagement by CD1d potentiates activation of $\mathrm{CD}^{+}$NKT cells $(15,16)$. Recent evidence suggests iNKT involvement in DENV pathogenesis, and the level of iNKT cell activation associates with the disease severity (17-19). Finally, another unconventional T cell subset, gamma delta $(\gamma \delta)$ $\mathrm{T}$ cells, has been shown to be able to kill dengue-infected cells and contribute to the immune response during DENV infection by providing an early source of IFN- $\gamma(20)$.

This review focuses on CD4 $\mathrm{T}$ cells that can also acquire a cytotoxic phenotype, which has been investigated by numerous studies over the past three decades (21). The ability of CD4 T cells to acquire cytotoxic functions have been mostly attributed to T helper type 1 (Th1) cells after viral infections; however, it is now clear that other CD4 $\mathrm{T}$ cell subsets including regulatory $\mathrm{T}$ (Treg) cells can also secrete effector molecules and exert cytotoxic effects $(22,23)$. Moreover, recent studies further suggest that cytotoxic CD4 T cells may represent a separate lineage independent of other CD4 T cell subsets and are induced by distinct environmental cues and transcriptional regulators, highlighting the versatility of CD4 T cell responses (24-26). Notably, cytotoxic CD4 $\mathrm{T}$ cells are readily detectable following DENV infection and correlate with enhanced protection against dengue disease $(12,27)$. We discuss the differentiation and function of cytotoxic CD4 T cells, especially in the context of DENV infection, and anticipate future studies into the therapeutic potentials of these intriguing cells in the development of anti-DENV vaccines and immunotherapies.

\section{CELLULAR AND ENVIRONMENTAL FACTORS THAT MEDIATE THE GENERATION OF CYTOTOXIC CD4 T CELLS}

The differentiation of diverse CD4 $\mathrm{T}$ cell subsets is induced and guided by antigens, costimulation, and distinct sets of cytokines, which are integrated to regulate the expression of transcription factors that are crucial for CD4 $\mathrm{T}$ cell lineage specification (28). In line with this notion, costimulatory signals mediated by OX40-OX40L and 4-1BB (29-31), as well as cytokines such as transforming growth factor- $\beta$ (TGF- $\beta$ ), type I interferons and IL-2 $(25,32-34)$, have been suggested to promote the differentiation of cytotoxic CD4 T cells. Although cytotoxic $\mathrm{CD} 4 \mathrm{~T}$ cells are often observed during chronic infections such as HIV, Epstein-Barr virus (EBV), human cytomegalovirus (HCMV), and mouse CMV (MCMV) infections (35-39), they are also readily detectable following acute lymphocytic choriomeningitis virus (LCMV), influenza virus, and ectromelia virus infections (40-44). Therefore, persistent antigenic stimulation may not be absolutely required for cytotoxic CD4 T cell differentiation. Furthermore, Brown et al. reported that IL-2 substantially enhances the cytotoxic functions of CD4 T cells that are activated with low antigen dose in vitro, suggesting that inflammatory cytokines may amplify $\mathrm{T}$ cell receptor (TCR) signals to promote the differentiation of cytotoxic CD4 T cells (32). Two additional common cytokine receptor $\gamma$-chain $\left(\gamma_{c}\right)$ family cytokines, IL-7 and IL-15, however, are dispensable for the formation of cytotoxic CD4 T cells, although IL-15 may promote their effector functions upon reactivation with TCR stimulus (24). Interestingly, IL-21, which is another member of the $\gamma_{c}$ family, has been shown to increase the production of the cytotoxic molecule granzyme B in CD8 T cells both in vivo and in vitro $(45,46)$. Thus, it would be interesting to investigate 
whether IL-21 also plays a role in the generation and/or functional maturation of cytotoxic CD4 T cells.

\section{MOLECULAR REGULATION OF CYTOTOXIC CD4 T CELL DIFFERENTIATION}

The integration and interpretation of numerous cellular and environmental parameters are mediated by transcriptional factors, and a number of transcriptional regulators have been implicated in the differentiation program of cytotoxic CD4 T cells (Figure 1). T-helper-inducing POZ/Kruppel-like factor (ThPOK) and Runt-related transcription factor 3 (Runx3), which suppress each other's expression, control the development of CD4 and CD8 T cells in the thymus, respectively (47). After exiting the thymus, mature CD4 T cells continue to express ThPOK, which suppresses Runx3 and maintains the lineage stability of CD4 $\mathrm{T}$ cells (48-50). Ectopic expression of ThPOK in CD8 T cells results in reduced expression of $\mathrm{CD} 8$, the T-box transcription factor eomesodermin (Eomes), as well as effector molecules such as
IFN- $\gamma$, granzyme B, and perforin, further supporting the notion that ThPOK restricts the initiation of cytotoxic T lymphocyte (CTL) differentiation program in CD4 T cells (51). In contrast, Runx3 promotes CD8 expression by binding its enhancer regions $(52,53)$ and also cooperates with Eomes and another T-box transcription factor, T-bet, to induce the manufacture of IFN- $\gamma$, perforin, and granzyme B $(54,55)$. Intriguingly, a portion of CD4 T cells downregulates their expression of ThPOK in the intestine, especially in the intraepithelial lymphocyte (IEL) compartment, under unimmunized conditions or following activation with their cognate antigen $(24,25)$. Conversely, these ThPOK ${ }^{\text {low }}$ CD4 T cells upregulate the expression of Runx3, thus resembling their CD8 T cell counterparts $(24,25)$. Consequently, these cells showed enhanced expression of cytotoxic effector lymphocytes-associated molecules, including 2B4, granzyme B, and CD107a, and demonstrated cytotoxicity in vitro $(24,25)$. It is further proposed that sustained antigenic stimulation and/or local environmental cues such as TGF- $\beta$ and retinoic acid (RA) induce the downregulation of ThPOK and the upregulation of Runx3, although the underlying signaling and transcriptional mechanisms are less well defined $(24,25)$. Thus, the antagonistic

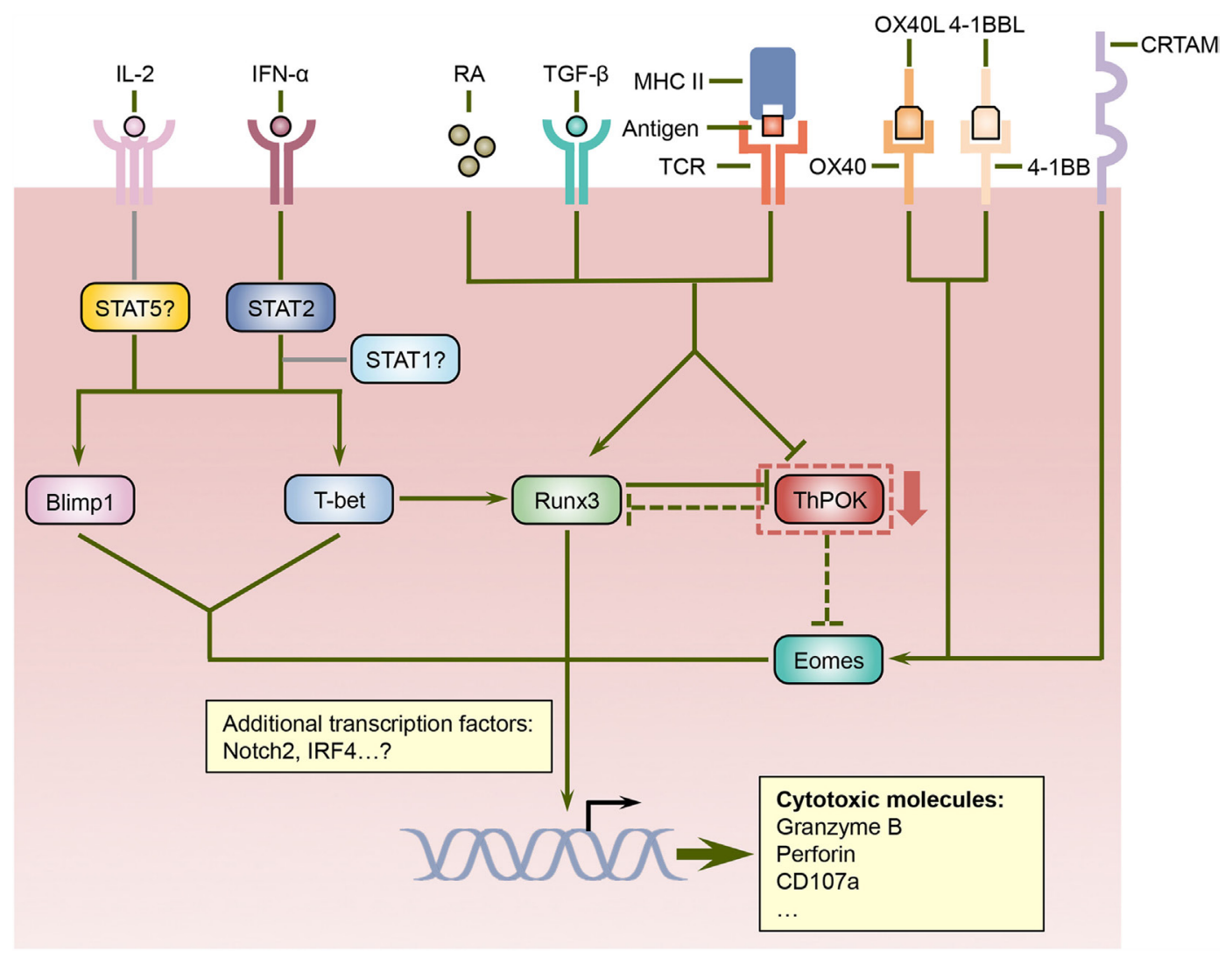

FIGURE 1 | Regulation of cytotoxic CD4 T cell differentiation by a network of signaling and transcriptional pathways. ThPOK is essential for the lineage specification and stability of CD4 helper T cells and counteracts Runx3, which coordinate the differentiation of CD8 T cells. Nevertheless, signals mediated by antigens, TGF- $\beta$, and retinoic acid (RA) can repress ThPOK expression in CD4 T cells, which results in elevated levels of Runx3. Since ThPOK inhibits Eomes, the suppression of ThPOK may also lead to increased Eomes expression. Furthermore, cytokines such as IL-2 and IFN- $\alpha$, costimulatory molecules including OX 40 and 4-1BB, as well as the transmembrane protein CRTAM can increase the expression and/or activities of Eomes as well as additional transcription factors such as Blimp1 and T-bet, which together with Runx3 direct the differentiation program of cytotoxic CD4 T cells. 
expression of ThPOK and Runx3 not only dictate the lineage stability of CD4 and CD8 T cells but also direct the establishment of cytotoxic CD4 T cells. Further studies are needed to determine whether and how the expression of ThPOK and Runx3 dictate the development of cytotoxic CD4 T cells following viral infections as well as the cellular and environmental factors that modulate their expression.

In addition to ThPOK and Runx3, several other transcription regulators have been suggested to regulate the differentiation of cytotoxic CD4 $\mathrm{T}$ cells. T-bet promotes the differentiation of effector CD8 T cells (56) and also induces the expression of Runx3 in CD4 T cells (54), suggesting that it may play a role in the formation of cytotoxic CD4 T cells. Indeed, T-betdeficient CD4 $\mathrm{T}$ cells demonstrated substantially reduced production of granzyme B following influenza virus infection (33). Furthermore, the acquisition of cytotoxic functions by CD4 $\mathrm{T}$ cells is also dependent upon B lymphocyte-induced maturation protein 1 (Blimp1), which facilitates the binding of T-bet to the granzyme B and perforin promoters (33). The upstream signals that drive the expression of T-bet and Blimp1 in CD4 T cells include IL- 2 and IFN- $\alpha$, and IFN- $\alpha$ may also exert its effects partially via signal transducer and activator of transcription 2 (STAT2) as evidenced by decreased T-bet and granzyme B expression in the absence of STAT2 (33). Since heterodimerization with activated STAT1 is required for the nuclear accumulation of phosphorylated STAT2 (57), whether STAT1 promotes cytotoxic CD4 T cell development in conjunction with STAT2 warrants further investigation. Surprisingly, STAT4, which has been shown to promote T-bet expression and Th1 cell differentiation in response to IL-12 signals (58-60), is dispensable for the upregulation of T-bet and granzyme B in CD4 T cells following influenza virus infection (33), indicating that cytotoxic CD4 T cells and Th1 cells may rely on distinct pathways for their differentiation. As discussed above, ThPOK suppresses the expression of Eomes, which cooperates with T-bet and Runx3 to promote the effector functions of CD8 T cells (55, 61-63), suggesting that Eomes may also participate in programing cytotoxic CD4 $\mathrm{T}$ cells when its expression is increased. Indeed, the engagement of costimulatory molecules OX40 and/or 4-1BB induces the expression of Eomes, which then upregulates the production of granzyme B by CD4 T cells and enhances their antitumor activities (29-31). In addition, a recent report shows that class I-restricted $\mathrm{T}$ cell-associated molecule (CRTAM) can promote the expression of Eomes and cytotoxic proteins including granzyme $\mathrm{B}$ and perforin in CD4 T cells (64). Therefore, Eomes may coordinate multiple signaling pathways to direct the development of cytotoxic CD4 $\mathrm{T}$ cells. Additional transcriptional regulators such as Notch2 (65), STAT5 (66), and interferon regulatory factor 4 (IRF4) (67) have been implicated in the manufacture of cytotoxic weaponry in CD8 T cells, and it would be interesting to investigate whether these transcriptional regulators also modulate the cytotoxic potential of CD4 T cells. In sum, the signals mediated by TCR, cytokines, costimulatory molecules, and other cell surface receptors are integrated and interpreted by a network of transcriptional regulators, which collectively orchestrate the differentiation of cytotoxic CD4 T cells.

\section{THE DEVELOPMENT OF CYTOTOXIC CD4 T CELLS FOLLOWING DENV INFECTIONS}

DENV-specific CD4 T cells with cytotoxic potential were initially observed with $\mathrm{T}$ cell clones isolated from a DENV-infected donor (68). These CD4 T cell clones demonstrate in vitro killing of target cells that display DENV antigens as assessed by chromium release assays, which are restricted by HLA class II molecules (68). Subsequent studies discovered that DENV non-structural (NS) proteins especially NS3 are the major targets of cytotoxic CD4 T cell clones and that many of these cell clones exhibit crossreactivity against several DENV serotypes (69-72). Thus, cytotoxic CD4 T cell may preferentially recognize conserved DENV antigens, and repeated antigenic stimulation may favor their formation. In addition to antigen-specific killing, anti-DENV cytotoxic CD4 T cell clones generated from DENV-immune donor can also mediate the lysis of non-antigen-presenting bystander target cells (73). While cytotoxic CD4 T cells lyse target cells pulsed with DENV antigens primarily via perforin-dependent mechanisms, the lysis of bystander target cells mainly relies on the Fas/Fas ligand (FasL) pathway (73). Additionally, a cytotoxic CD4 $\mathrm{T}$ cell clone has also been isolated from DENV-infected mice and is able to kill DENV antigen-pulsed target cells in vitro (74). Furthermore, Yauch et al. showed, using a mouse model of DENV infection, that CD4 T cells can mediate DENV-specific killing of target cells in vivo, although the production of cytotoxic molecules by CD4 T cells was not assessed (75). Immunization with CD4 $\mathrm{T}$ cell epitopes derived from DENV NS proteins NS2B and NS3 can accelerate viral clearance following DENV challenge, suggesting that the induction of cytotoxic CD4 T cells by vaccination may be beneficial for the control of secondary infections with DENV (75).

Previous studies have demonstrated that CD4 T cells with cytotoxic potential as assessed by the expression of CD107a are present in patients associated with both primary and secondary DENV infections, although the frequencies of these cells vary according to infection history and disease severity (12). Interestingly, the frequency of DENV-specific CD107a ${ }^{+}$CD4 T cells is higher in DF patients compared with those who had a more severe form of the disease, DHF, implicating a protective role for cytotoxic CD4 $\mathrm{T}$ cells in DENV-infected patients (12). Our laboratory has recently discovered that a subset of CD4 T cells expand as a function of DENV infection history and is most prominently represented in donors associated with multiple DENV infections (27). These CD4 T cells display a CD45RA ${ }^{\text {high }} C C R 7^{\text {low }}$ phenotype, which is distinct from their CD45RA ${ }^{\text {low }}$ CCR7 ${ }^{\text {high }}$ central memory $\mathrm{T}$ (Tcm) and CD45RA ${ }^{\text {low } C C R 7}{ }^{\text {low }}$ effector memory $\mathrm{T}$ (Tem) counterparts, and thus are designated effector memory RA T (Temra) cells. Compared with CD4 Tcm or Tem cells, a higher proportion of CD4 Temra cells express CD8 $\alpha$, the degranulation marker CD107a, as well as other cytotoxic effector molecules such as granzyme B and perforin, suggesting that CD4 Temra population contains anti-DENV cytotoxic CD4 T cells (27). Additionally, CD4 Temra cells also had increased expression of CD226 (27), which is a costimulatory molecule that has been shown to enhance the effector and cytotoxic functions of CD8 $\mathrm{T}$ cells $(76,77)$. Conversely, these cells downregulate CD28 
expression (19), which is consistent with previous observations that $\mathrm{CD} 4^{+} \mathrm{CD} 28^{-} \mathrm{T}$ cells are associated with enhanced cytotoxic functions following infections with $\mathrm{CMV}$ and hepatitis $\mathrm{B}$ virus ( $\mathrm{HBV})(78,79)$. As discussed earlier, the T-box transcription factors T-bet and Eomes coordinate the development of cytotoxic CD4 T cells. Notably, the vast majority of CD4 Temra cells express high levels of T-bet and Eomes, further supporting the notion that CD4 Temra cells encompass cytotoxic CD4 T cell subset in terms of their phenotypic and functional attributes, as well as their transcriptional signatures. Since cytotoxic CD4 Temra cells are generally detected following secondary DENV infections, these findings further support that DENV-specific cytotoxic CD4 $\mathrm{T}$ cells are induced by repeated TCR stimulation from conserved DENV antigens, which is consistent with in vitro studies using cytotoxic CD4 T cell clones. Intriguingly, CD4 Temra cells with cytotoxic potential observed in secondary DENV-infected donors phenotypically and functionally resemble the live attenuated yellow fever vaccine 17D (YF-17D)-elicited CD8 Temra cells, which are highly proliferative and polyfunctional as evidenced by their ability to produce various cytokines and cytotoxic molecules including CD107a (80). Since vaccination with YF-17D has been tremendously successful in controlling yellow fever virus (81), Temra phenotype cytotoxic CD4 T cells may be highly relevant in vaccine-elicited protection against DENV infection.

The majority of DENV-specific CD4 Temra cells are not associated with the phenotypes of Th1, Th2, or Th17 cells as evidenced by their lack of expression of the chemokine receptors CXCR3, CCR4, and CCR6, which have been used to distinguish between these distinct CD4 T cell subsets $(27,82)$. Since CD4 Temra cells encompass cytotoxic populations, these findings further support the notion that cytotoxic CD4 T cells may represent an independent CD4 T cell lineage. Notably, Temra cells upregulate the expression of the chemokine receptor CX3CR1, which binds to CX3CL1 (fractalkine) and has been implicated in promoting the adhesion and migration of CD8 $\mathrm{T}$ cells $(83,84)$. Thus, CX3CR1 may facilitate the trafficking of DENV-specific CD4 $\mathrm{T}$ cells, particularly cytotoxic CD4 T cells, to infected tissues. Intriguingly, accumulating studies have demonstrated that CD4 tissue-resident memory $\mathrm{T}$ ( $\mathrm{Trm}$ ) cells reside in sites of pathogen entry and are crucial for the control of viral pathogens such as influenza virus and herpes simplex virus by providing immediate effector functions $(85,86)$. Therefore, it would be interesting to investigate whether DENV-specific CD4 Trm cells develop following infections and whether CX3CR1 plays a role in their establishment and maintenance in non-lymphoid tissues such as the skin. Importantly, the expression of CX3CR1 is also associated with the cytotoxic functions of CD4 T cells as isolated CX3CR $1^{+}$CD4 $\mathrm{T}$ cells demonstrated specific killing of DENV epitopes-pulsed target cells ex vivo (27). This is consistent with a recent report showing that $\mathrm{CX} 3 \mathrm{CR} 1^{+} \mathrm{CD} 8 \mathrm{~T}$ cells have potent cytotoxic functions and express elevated levels of granzyme $\mathrm{B}$ and perforin (87). Thus, CX3CR1 ${ }^{+}$cytotoxic CD4 T cells may play an important role in viral control by directly targeting DENVinfected cells in tissues.

\section{CYTOTOXIC CD4 T CELLS CORRELATE WITH HLA-ASSOCIATED PROTECTION AGAINST DENV INFECTION}

Additional insights into the question of whether cytotoxic CD4 $\mathrm{T}$ cells are beneficial in the context of DENV infections come

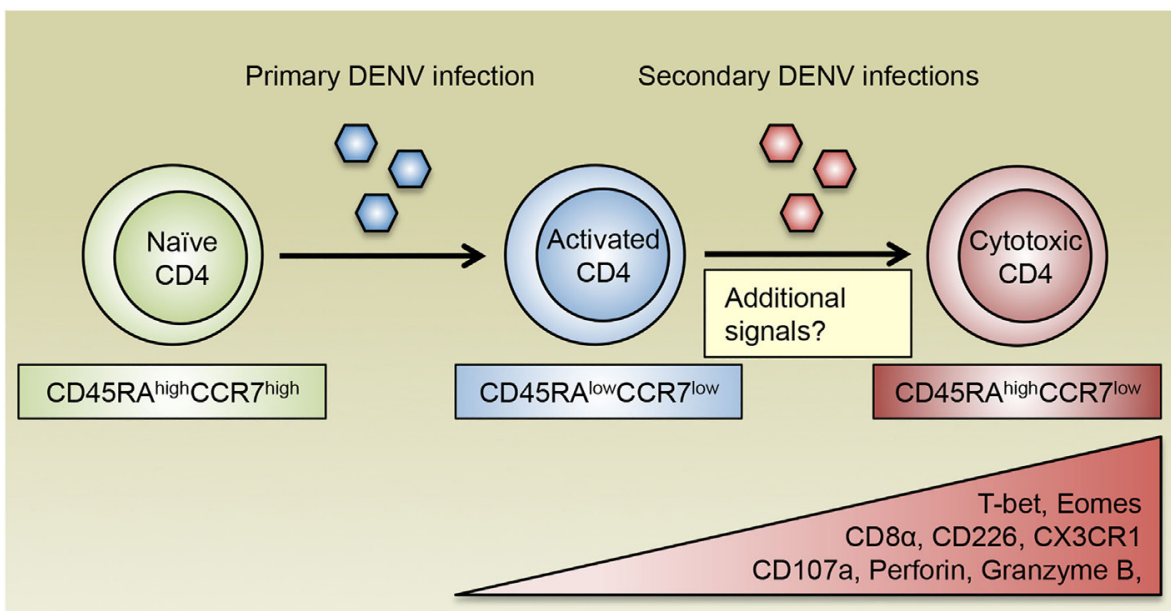

FIGURE 2 | The generation of cytotoxic CD4 T cells following DENV infections. DENV-specific naïve CD4 T cells are activated during primary DENV infection and acquire an effector phenotype and the ability to produce inflammatory cytokines such as IFN- $\gamma$. Following reexposure to secondary heterologous infections, DENV-specific CD4 T cells receive repeated antigenic signals and differentiate into cytotoxic CD4 T cells, which display a CD45RA high CCR7 low Temra phenotype and are characterized by their expression of the chemokine receptor CX3CR1. Cytotoxic CD4 T cells also upregulate the expression of CD8 $\alpha$ and CD226, as well as the transcription factors T-bet and Eomes, which may cooperate with additional transcription regulators to induce the production of cytotoxic molecules such as CD107a, perforin, and granzyme B. In addition to recurring antigens, costimulatory molecules, cytokines, and other environmental cues are all likely to modulate the differentiation of cytotoxic CD4 T cells. 
from the association of HLA allelic variants with relative susceptibility or resistance to severe DENV-associated diseases. For instance, HLA class II molecules DRB1*04:01 and DRB1*08:02 are associated with resistance and susceptibility to severe DENV diseases, respectively (88-90). Intriguingly, the frequency of DENV-specific CD4 Temra cells is higher in donors expressing the protective allele $\mathrm{DRB} 1^{\star} 04: 01$ compared with those expressing the susceptible allele DRB1*08:02. Moreover, CD4 Temra cells restricted by $\mathrm{DRB} 1^{\star} 04: 01$ express substantially higher levels of cytotoxic proteins including CD107a, perforin, and granzyme $\mathrm{B}$ compared with their $\mathrm{DRB} 1^{\star} 08: 02$-restricted counterparts. Thus, increased abundance and functionality of cytotoxic CD4 Temra cells may be associated with enhanced protection against severe dengue disease. Furthermore, the capacity of CX3CR1 ${ }^{+}$CD 4 $T$ cells to kill target cells varies according to HLA restrictions with the protective allele $\mathrm{DRB} 1^{\star} 04: 01$-restricted cells showing higher cytotoxic activities than their susceptible allele DRB1*08:02restricted counterparts, which again indicates that cytotoxic CD4 $\mathrm{T}$ cell responses correlate with protection from severe dengue disease. Since DENV primarily infects major histocompatibility complex (MHC) class II-expressing antigen-presenting cells such as monocytes, macrophages, and DCs $(91,92)$, which could be exacerbated by antibody-dependent enhancement (ADE) mechanism (93), cytotoxic CD4 T cells may play important roles in controlling the spread of DENV infection by directly eliminating these cells. Other cell types such as epithelial cells can be induced to upregulate the expression of MHC class II molecules following viral infection and potentially become additional targets for cytotoxic CD4 T cells (43). Therefore, cytotoxic CD4 T cells may contribute to HLA-associated protection against DENV infection by targeting DENV-infected cells of various types. Taken together, we propose a model where repeated antigenic signals as well as other potential cellular and environmental stimuli facilitate the formation of DENV-specific cytotoxic CD4 T cells, which exhibit a Temra phenotype with upregulated CX3CR1 expression and mediate protective responses against DENV infection (Figure 2).

\section{CONCLUSION AND PERSPECTIVE}

Despite the recent approval of Dengvaxia ${ }^{\circledR}$ in several countries where DENV is epidemic, our need for an efficacious DENV vaccine is still unsatisfied. Accumulating studies strongly indicate that, in addition to CD8 $\mathrm{T}$ cells, cytotoxic CD4 T cells may

\section{REFERENCES}

1. Bhatt S, Gething PW, Brady OJ, Messina JP, Farlow AW, Moyes CL, et al. The global distribution and burden of dengue. Nature (2013) 496:504-7. doi: $10.1038 /$ nature 12060

2. Halstead SB. Pathogenesis of dengue: challenges to molecular biology. Science (1988) 239:476-81. doi:10.1126/science.3277268

3. Alagarasu K. Introducing dengue vaccine: implications for diagnosis in dengue vaccinated subjects. Vaccine (2016) 34:2759-61. doi:10.1016/ j.vaccine.2016.04.070

4. Sabchareon A, Wallace D, Sirivichayakul C, Limkittikul K, Chanthavanich P, Suvannadabba S, et al. Protective efficacy of the recombinant, live-attenuated, CYD tetravalent dengue vaccine in Thai schoolchildren: a randomised, controlled phase 2b trial. Lancet (2012) 380:1559-67. doi:10.1016/S0140-6736 (12)61428-7 play an important role in eliminating DENV-infected cells and protecting the hosts from severe dengue disease. Since the generation of cytotoxic CD4 T cells is concurrent with multiple DENV infections, it would be interesting to identify the TCR-specificity of cytotoxic CD4 T cells and confirm whether they respond to conserved epitopes shared by different DENV serotypes. This would allow for the design of vaccines that include such epitopes and preferentially induce the formation of cytotoxic CD4 T cells. In addition to vaccines, adoptive transfer of engineered CD4 $\mathrm{T}$ cells that are specific for such antigens may accelerate viral clearance and benefit the treatment of DENV infection, as clinical trials of T cells that are engineered to express transgenic TCRs or chimeric antigen receptors (CARs) have generated promising results in treating cancers (94). Furthermore, costimulatory molecules and cytokines that are involved in the differentiation and function of cytotoxic CD4 T cells may potentially be used as adjuvants to enhance the cytotoxic effects of DENV-specific CD4 T cells. Additionally, elucidating the factors that control the development of cytotoxic CD4 T cells may allow one to manipulate their expression, availability, and activity in order to reshape CD4 T cell responses in patients expressing susceptible alleles and redirect antiviral CD4 $\mathrm{T}$ cells to differentiate into potent cytotoxic cells. Finally, $\mathrm{T}$ cell differentiation and function is greatly influenced by nutrients and metabolism (95). For instance, glucose and glycolysis promote the expression of cytotoxic molecules in CD8 T cells (96). Thus, modulation of the metabolic program may provide additional opportunities to enhance cytotoxic CD4 T cell response. In summary, future investigations into the antigenic, environmental, and cellular parameters that configure the formation, migration, and maintenance of cytotoxic CD4 T cells may reveal novel strategies for developing and improving vaccines and therapies that fight DENV as well as other emerging pathogens.

\section{AUTHOR CONTRIBUTIONS}

YT wrote the manuscript; YT and DW designed the figures; AS and DW critically edited the manuscript.

\section{FUNDING}

This work was supported by National Institutes of Health (contracts Nr. HHSN272200900042C and HHSN27220140045C).

5. Villar L, Dayan GH, Arredondo-Garcia JL, Rivera DM, Cunha R, Deseda $\mathrm{C}$, et al. Efficacy of a tetravalent dengue vaccine in children in Latin America. N Engl JMed (2015) 372:113-23. doi:10.1056/NEJMoa 1411037

6. Capeding MR, Tran NH, Hadinegoro SR, Ismail HI, Chotpitayasunondh T, Chua MN, et al. Clinical efficacy and safety of a novel tetravalent dengue vaccine in healthy children in Asia: a phase 3, randomised, observer-masked, placebo-controlled trial. Lancet (2014) 384:1358-65. doi:10.1016/ S0140-6736(14)61060-6

7. Hadinegoro SR, Arredondo-Garcia JL, Capeding MR, Deseda C, Chotpitayasunondh T, Dietze R, et al. Efficacy and long-term safety of a dengue vaccine in regions of endemic disease. N Engl J Med (2015) 373:1195-206. doi:10.1056/NEJMoa1506223

8. Weiskopf D, Sette A. T-cell immunity to infection with dengue virus in humans. Front Immunol (2014) 5:93. doi:10.3389/fimmu.2014.00093 
9. Gunther VJ, Putnak R, Eckels KH, Mammen MP, Scherer JM, Lyons A, et al. A human challenge model for dengue infection reveals a possible protective role for sustained interferon gamma levels during the acute phase of illness. Vaccine (2011) 29:3895-904. doi:10.1016/j.vaccine.2011.03.038

10. Hatch S, Endy TP, Thomas S, Mathew A, Potts J, Pazoles P, et al. Intracellular cytokine production by dengue virus-specific $\mathrm{T}$ cells correlates with subclinical secondary infection. J Infect Dis (2011) 203:1282-91. doi:10.1093/infdis/ jir012

11. Weiskopf D, Angelo MA, de Azeredo EL, Sidney J, Greenbaum JA, Fernando AN, et al. Comprehensive analysis of dengue virus-specific responses supports an HLA-linked protective role for CD8+ T cells. Proc Natl Acad Sci U S A (2013) 110:E2046-53. doi:10.1073/pnas.1305227110

12. Duangchinda T, Dejnirattisai W, Vasanawathana S, Limpitikul W, Tangthawornchaikul N, Malasit P, et al. Immunodominant T-cell responses to dengue virus NS3 are associated with DHF. Proc Natl Acad Sci U S A (2010) 107:16922-7. doi:10.1073/pnas.1010867107

13. Libraty DH, Pichyangkul S, Ajariyakhajorn C, Endy TP, Ennis FA. Human dendritic cells are activated by dengue virus infection: enhancement by gamma interferon and implications for disease pathogenesis. J Virol (2001) 75:3501-8. doi:10.1128/JVI.75.8.3501-3508.2001

14. Bendelac A. CD1: presenting unusual antigens to unusual $\mathrm{T}$ lymphocytes. Science (1995) 269:185-6. doi:10.1126/science.7542402

15. Liao CM, Zimmer MI, Wang CR. The functions of type I and type II natural killer $\mathrm{T}$ cells in inflammatory bowel diseases. Inflamm Bowel Dis (2013) 19:1330-8. doi:10.1097/MIB.0b013e318280b1e3

16. Thedrez A, de Lalla C, Allain S, Zaccagnino L, Sidobre S, Garavaglia C, et al. CD4 engagement by CD1d potentiates activation of CD4+ invariant NKT cells. Blood (2007) 110:251-8. doi:10.1182/blood-2007-01-066217

17. Matangkasombut P, Chan-In W, Opasawaschai A, Pongchaikul P, Tangthawornchaikul N, Vasanawathana S, et al. Invariant NKT cell response to dengue virus infection in human. PLoS Negl Trop Dis (2014) 8:e2955. doi:10.1371/journal.pntd.0002955

18. Renneson J, Guabiraba R, Maillet I, Marques RE, Ivanov S, Fontaine J, et al. A detrimental role for invariant natural killer $\mathrm{T}$ cells in the pathogenesis of experimental dengue virus infection. Am J Pathol (2011) 179:1872-83. doi:10.1016/j.ajpath.2011.06.023

19. St John AL, Rathore AP, Yap H, Ng ML, Metcalfe DD, Vasudevan SG, et al. Immune surveillance by mast cells during dengue infection promotes natural killer (NK) and NKT-cell recruitment and viral clearance. Proc Natl Acad Sci US A (2011) 108:9190-5. doi:10.1073/pnas.1105079108

20. Tsai CY, Liong KH, Gunalan MG, Li N, Lim DS, Fisher DA, et al. Type I IFNs and IL-18 regulate the antiviral response of primary human gammadelta $\mathrm{T}$ cells against dendritic cells infected with dengue virus. J Immunol (2015) 194:3890-900. doi:10.4049/jimmunol.1303343

21. Marshall NB, Swain SL. Cytotoxic CD4 T cells in antiviral immunity. J Biomed Biotechnol (2011) 2011:954602. doi:10.1155/2011/954602

22. Grossman WJ, Verbsky JW, Barchet W, Colonna M, Atkinson JP, Ley TJ. Human $\mathrm{T}$ regulatory cells can use the perforin pathway to cause autologous target cell death. Immunity (2004) 21:589-601. doi:10.1016/j.immuni.2004.09.002

23. Cao X, Cai SF, Fehniger TA, Song J, Collins LI, Piwnica-Worms DR, et al. Granzyme B and perforin are important for regulatory $\mathrm{T}$ cell-mediated suppression of tumor clearance. Immunity (2007) 27:635-46. doi:10.1016/ j.immuni.2007.08.014

24. Mucida D, Husain MM, Muroi S, van Wijk F, Shinnakasu R, Naoe Y, et al. Transcriptional reprogramming of mature CD4(+) helper T cells generates distinct MHC class II-restricted cytotoxic T lymphocytes. Nat Immunol (2013) 14:281-9. doi:10.1038/ni.2523

25. Reis BS, Rogoz A, Costa-Pinto FA, Taniuchi I, Mucida D. Mutual expression of the transcription factors Runx3 and ThPOK regulates intestinal CD4(+) T cell immunity. Nat Immunol (2013) 14:271-80. doi:10.1038/ni.2518

26. Cheroutre H, Husain MM. CD4 CTL: living up to the challenge. Semin Immunol (2013) 25:273-81. doi:10.1016/j.smim.2013.10.022

27. Weiskopf D, Bangs DJ, Sidney J, Kolla RV, De Silva AD, de Silva AM, et al. Dengue virus infection elicits highly polarized CX3CR1+ cytotoxic CD4+ T cells associated with protective immunity. Proc Natl Acad Sci U S A (2015) 112:E4256-63. doi:10.1073/pnas.1505956112

28. Tripathi SK, Lahesmaa R. Transcriptional and epigenetic regulation of T-helper lineage specification. Immunol Rev (2014) 261:62-83. doi:10.1111/ imr. 12204
29. Curran MA, Geiger TL, Montalvo W, Kim M, Reiner SL, Al-Shamkhani A, et al. Systemic 4-1BB activation induces a novel $\mathrm{T}$ cell phenotype driven by high expression of eomesodermin. J Exp Med (2013) 210:743-55. doi:10.1084/ jem.20121190

30. Hirschhorn-Cymerman D, Budhu S, Kitano S, Liu C, Zhao F, Zhong H, et al. Induction of tumoricidal function in CD4+ T cells is associated with concomitant memory and terminally differentiated phenotype. J Exp Med (2012) 209:2113-26. doi:10.1084/jem.20120532

31. Qui HZ, Hagymasi AT, Bandyopadhyay S, St Rose MC, Ramanarasimhaiah R, Menoret A, et al. CD134 plus CD137 dual costimulation induces eomesodermin in CD4 T cells to program cytotoxic Th1 differentiation. J Immunol (2011) 187:3555-64. doi:10.4049/jimmunol.1101244

32. Brown DM, Kamperschroer C, Dilzer AM, Roberts DM, Swain SL. IL-2 and antigen dose differentially regulate perforin- and FasL-mediated cytolytic activity in antigen specific CD4+ T cells. Cell Immunol (2009) 257:69-79. doi:10.1016/j.cellimm.2009.03.002

33. Hua L, Yao S, Pham D, Jiang L, Wright J, Sawant D, et al. Cytokine-dependent induction of CD4+ T cells with cytotoxic potential during influenza virus infection. J Virol (2013) 87:11884-93. doi:10.1128/JVI.01461-13

34. Workman AM, Jacobs AK, Vogel AJ, Condon S, Brown DM. Inflammation enhances IL-2 driven differentiation of cytolytic CD4 T cells. PLoS One (2014) 9:e89010. doi:10.1371/journal.pone.0089010

35. Appay V, Zaunders JJ, Papagno L, Sutton J, Jaramillo A, Waters A, et al. Characterization of CD4(+) CTLs ex vivo. J Immunol (2002) 168:5954-8. doi:10.4049/jimmunol.168.11.5954

36. Casazza JP, Betts MR, Price DA, Precopio ML, Ruff LE, Brenchley JM, et al. Acquisition of direct antiviral effector functions by CMV-specific CD4+ T lymphocytes with cellular maturation. J Exp Med (2006) 203:2865-77. doi:10.1084/jem.20052246

37. Suni MA, Ghanekar SA, Houck DW, Maecker HT, Wormsley SB, Picker LJ, et al. CD4(+)CD8(dim) T lymphocytes exhibit enhanced cytokine expression, proliferation and cytotoxic activity in response to HCMV and HIV-1 antigens. Eur JImmunol (2001) 31:2512-20. doi:10.1002/1521-4141(200108)31:8<2512::AID-IMMU2512>3.0.CO;2-M

38. Verma S, Weiskopf D, Gupta A, McDonald B, Peters B, Sette A, et al. Cytomegalovirus-specific CD4 $\mathrm{T}$ cells are cytolytic and mediate vaccine protection. J Virol (2016) 90:650-8. doi:10.1128/JVI.02123-15

39. Haigh TA, Lin X, Jia H, Hui EP, Chan AT, Rickinson AB, et al. EBV latent membrane proteins (LMPs) 1 and 2 as immunotherapeutic targets: LMPspecific CD4+ cytotoxic T cell recognition of EBV-transformed B cell lines. J Immunol (2008) 180:1643-54. doi:10.4049/jimmunol.180.3.1643

40. Zajac AJ, Quinn DG, Cohen PL, Frelinger JA. Fas-dependent CD4+ cytotoxic T-cell-mediated pathogenesis during virus infection. Proc Natl Acad Sci U S A (1996) 93:14730-5. doi:10.1073/pnas.93.25.14730

41. Jellison ER, Kim SK, Welsh RM. Cutting edge: MHC class II-restricted killing in vivo during viral infection. J Immunol (2005) 174:614-8. doi:10.4049/ jimmunol.174.2.614

42. Brown DM, Lee S, Garcia-Hernandez Mde L, Swain SL. Multifunctional CD4 cells expressing gamma interferon and perforin mediate protection against lethal influenza virus infection. J Virol (2012) 86:6792-803. doi:10.1128/ JVI.07172-11

43. Wilkinson TM, Li CK, Chui CS, Huang AK, Perkins M, Liebner JC, et al. Preexisting influenza-specific CD4+ $\mathrm{T}$ cells correlate with disease protection against influenza challenge in humans. Nat Med (2012) 18:274-80. doi:10.1038/nm.2612

44. Fang M, Siciliano NA, Hersperger AR, Roscoe F, Hu A, Ma X, et al. Perforindependent CD4+ T-cell cytotoxicity contributes to control a murine poxvirus infection. Proc Natl Acad Sci U S A (2012) 109:9983-8. doi:10.1073/ pnas. 1202143109

45. Tian Y, Cox MA, Kahan SM, Ingram JT, Bakshi RK, Zajac AJ. A context-dependent role for IL-21 in modulating the differentiation, distribution, and abundance of effector and memory CD8 T cell subsets. J Immunol (2016) 196:2153-66. doi:10.4049/jimmunol.1401236

46. Sutherland AP, Joller N, Michaud M, Liu SM, Kuchroo VK, Grusby MJ. IL-21 promotes CD8+ CTL activity via the transcription factor T-bet. J Immunol (2013) 190:3977-84. doi:10.4049/jimmunol.1201730

47. Egawa T. Regulation of CD4 and CD8 coreceptor expression and CD4 versus CD8 lineage decisions. Adv Immunol (2015) 125:1-40. doi:10.1016/ bs.ai.2014.09.001 
48. Wang L, Wildt KF, Castro E, Xiong Y, Feigenbaum L, Tessarollo L, et al. The zinc finger transcription factor Zbtb7b represses CD8-lineage gene expression in peripheral CD4+ T cells. Immunity (2008) 29:876-87. doi:10.1016/ j.immuni.2008.09.019

49. Egawa T, Littman DR. ThPOK acts late in specification of the helper $\mathrm{T}$ cell lineage and suppresses Runx-mediated commitment to the cytotoxic $\mathrm{T}$ cell lineage. Nat Immunol (2008) 9:1131-9. doi:10.1038/ni.1652

50. Vacchio MS, Wang L, Bouladoux N, Carpenter AC, Xiong Y, Williams LC, et al. A ThPOK-LRF transcriptional node maintains the integrity and effector potential of post-thymic CD4+ T cells. Nat Immunol (2014) 15:947-56. doi:10.1038/ni.2960

51. Jenkinson SR, Intlekofer AM, Sun G, Feigenbaum L, Reiner SL, Bosselut R. Expression of the transcription factor cKrox in peripheral CD8 $\mathrm{T}$ cells reveals substantial postthymic plasticity in CD4-CD8 lineage differentiation. J Exp Med (2007) 204:267-72. doi:10.1084/jem.20061982

52. Sato T, Ohno S, Hayashi T, Sato C, Kohu K, Satake M, et al. Dual functions of Runx proteins for reactivating CD8 and silencing CD4 at the commitment process into CD8 thymocytes. Immunity (2005) 22:317-28. doi:10.1016/ j.immuni.2005.01.012

53. Hassan H, Sakaguchi S, Tenno M, Kopf A, Boucheron N, Carpenter AC, et al. $\mathrm{Cd} 8$ enhancer E8I and Runx factors regulate CD8alpha expression in activated CD8+ T cells. Proc Natl Acad Sci U S A (2011) 108:18330-5. doi:10.1073/ pnas. 1105835108

54. Djuretic IM, Levanon D, Negreanu V, Groner Y, Rao A, Ansel KM. Transcription factors T-bet and Runx 3 cooperate to activate Ifng and silence Il4 in T helper type 1 cells. Nat Immunol (2007) 8:145-53. doi:10.1038/ni1424

55. Cruz-Guilloty F, Pipkin ME, Djuretic IM, Levanon D, Lotem J, Lichtenheld MG, et al. Runx3 and T-box proteins cooperate to establish the transcriptional program of effector CTLs. J Exp Med (2009) 206:51-9. doi:10.1084/ jem.20081242

56. Joshi NS, Cui W, Chandele A, Lee HK, Urso DR, Hagman J, et al. Inflammation directs memory precursor and short-lived effector $\mathrm{CD} 8(+) \mathrm{T}$ cell fates via the graded expression of T-bet transcription factor. Immunity (2007) 27:281-95. doi:10.1016/j.immuni.2007.07.010

57. Banninger G, Reich NC. STAT2 nuclear trafficking. J Biol Chem (2004) 279:39199-206. doi:10.1074/jbc.M400815200

58. Schulz EG, Mariani L, Radbruch A, Hofer T. Sequential polarization and imprinting of type $1 \mathrm{~T}$ helper lymphocytes by interferon-gamma and interleukin-12. Immunity (2009) 30:673-83. doi:10.1016/j.immuni.2009.03.013

59. Thieu VT, Yu Q, Chang HC, Yeh N, Nguyen ET, Sehra S, et al. Signal transducer and activator of transcription 4 is required for the transcription factor T-bet to promote T helper 1 cell-fate determination. Immunity (2008) 29:679-90. doi:10.1016/j.immuni.2008.08.017

60. Zhu J, Jankovic D, Oler AJ, Wei G, Sharma S, Hu G, et al. The transcription factor T-bet is induced by multiple pathways and prevents an endogenous Th2 cell program during Th1 cell responses. Immunity (2012) 37:660-73. doi:10.1016/j.immuni.2012.09.007

61. Pearce EL, Mullen AC, Martins GA, Krawczyk CM, Hutchins AS, Zediak VP, et al. Control of effector CD8+ T cell function by the transcription factor eomesodermin. Science (2003) 302:1041-3. doi:10.1126/science.1090148

62. Intlekofer AM, Takemoto N, Wherry EJ, Longworth SA, Northrup JT, Palanivel VR, et al. Effector and memory CD8+ T cell fate coupled by T-bet and eomesodermin. Nat Immunol (2005) 6:1236-44. doi:10.1038/ni1268

63. Pipkin ME, Sacks JA, Cruz-Guilloty F, Lichtenheld MG, Bevan MJ, Rao A. Interleukin-2 and inflammation induce distinct transcriptional programs that promote the differentiation of effector cytolytic T cells. Immunity (2010) 32:79-90. doi:10.1016/j.immuni.2009.11.012

64. Takeuchi A, Badr Mel S, Miyauchi K, Ishihara C, Onishi R, Guo Z, et al. CRTAM determines the CD4+ cytotoxic T lymphocyte lineage. J Exp Med (2016) 213:123-38. doi:10.1084/jem.20150519

65. Maekawa Y, Minato Y, Ishifune C, Kurihara T, Kitamura A, Kojima H, et al. Notch2 integrates signaling by the transcription factors RBP-J and CREB1 to promote T cell cytotoxicity. Nat Immunol (2008) 9:1140-7. doi:10.1038/ ni. 1649

66. Verdeil G, Puthier D, Nguyen C, Schmitt-Verhulst AM, Auphan-Anezin N. STAT5-mediated signals sustain a TCR-initiated gene expression program toward differentiation of CD8 T cell effectors. J Immunol (2006) 176:4834-42. doi:10.4049/jimmunol.176.8.4834
67. Raczkowski F, Ritter J, Heesch K, Schumacher V, Guralnik A, Hocker L, et al. The transcription factor interferon regulatory factor 4 is required for the generation of protective effector CD8+ T cells. Proc Natl Acad Sci U S A (2013) 110:15019-24. doi:10.1073/pnas.1309378110

68. Kurane I, Meager A, Ennis FA. Dengue virus-specific human T cell clones. Serotype crossreactive proliferation, interferon gamma production, and cytotoxic activity. J Exp Med (1989) 170:763-75. doi:10.1084/jem.170.3.763

69. Kurane I, Brinton MA, Samson AL, Ennis FA. Dengue virus-specific, human CD4+ CD8- cytotoxic T-cell clones: multiple patterns of virus cross-reactivity recognized by NS3-specific T-cell clones. J Virol (1991) 65:1823-8.

70. Zeng L, Kurane I, Okamoto Y, Ennis FA, Brinton MA. Identification of amino acids involved in recognition by dengue virus NS3-specific, HLA-DR15restricted cytotoxic CD4+ T-cell clones. J Virol (1996) 70:3108-17.

71. Kurane I, Zeng L, Brinton MA, Ennis FA. Definition of an epitope on NS3 recognized by human $\mathrm{CD} 4+$ cytotoxic $\mathrm{T}$ lymphocyte clones cross-reactive for dengue virus types 2, 3, and 4. Virology (1998) 240:169-74. doi:10.1006/ viro.1997.8925

72. Mathew A, Kurane I, Green S, Stephens HA, Vaughn DW, Kalayanarooj S, et al. Predominance of HLA-restricted cytotoxic T-lymphocyte responses to serotype-cross-reactive epitopes on nonstructural proteins following natural secondary dengue virus infection. J Virol (1998) 72:3999-4004.

73. Gagnon SJ, Ennis FA, Rothman AL. Bystander target cell lysis and cytokine production by dengue virus-specific human $\mathrm{CD} 4(+)$ cytotoxic T-lymphocyte clones. J Virol (1999) 73:3623-9.

74. Rothman AL, Kurane I, Ennis FA. Multiple specificities in the murine CD4+ and CD8+ T-cell response to dengue virus. J Virol (1996) 70:6540-6.

75. Yauch LE, Prestwood TR, May MM, Morar MM, Zellweger RM, Peters B, et al. CD4+ T cells are not required for the induction of dengue virus-specific $\mathrm{CD} 8+\mathrm{T}$ cell or antibody responses but contribute to protection after vaccination. J Immunol (2010) 185:5405-16. doi:10.4049/jimmunol.1001709

76. Ramsbottom KM, Hawkins ED, Shimoni R, McGrath M, Chan CJ, Russell SM, et al. Cutting edge: DNAX accessory molecule 1-deficient CD8+ T cells display immunological synapse defects that impair antitumor immunity. J Immunol (2014) 192:553-7. doi:10.4049/jimmunol.1302197

77. Cella M, Presti R, Vermi W, Lavender K, Turnbull E, Ochsenbauer-Jambor C, et al. Loss of DNAM-1 contributes to CD8+ T-cell exhaustion in chronic HIV-1 infection. Eur J Immunol (2010) 40:949-54. doi:10.1002/ eji.200940234

78. van Leeuwen EM, Remmerswaal EB, Vossen MT, Rowshani AT, Wertheimvan Dillen PM, van Lier RA, et al. Emergence of a CD4+CD28- granzyme $\mathrm{B}+$, cytomegalovirus-specific $\mathrm{T}$ cell subset after recovery of primary cytomegalovirus infection. J Immunol (2004) 173:1834-41. doi:10.4049/ jimmunol.173.3.1834

79. Wang Y, Bai J, Li F, Wang H, Fu X, Zhao T, et al. Characteristics of expanded $\mathrm{CD} 4+\mathrm{CD} 28$ null $\mathrm{T}$ cells in patients with chronic hepatitis B. Immunol Invest (2009) 38:434-46. doi:10.1080/08820130902943105

80. Akondy RS, Monson ND, Miller JD, Edupuganti S, Teuwen D, Wu H, et al. The yellow fever virus vaccine induces a broad and polyfunctional human memory CD8+ T cell response. J Immunol (2009) 183:7919-30. doi:10.4049/ jimmunol.0803903

81. Barnett ED. Yellow fever: epidemiology and prevention. Clin Infect Dis (2007) 44:850-6. doi:10.1086/511869

82. Sallusto F, Lanzavecchia A. Heterogeneity of CD4+ memory T cells: functional modules for tailored immunity. Eur J Immunol (2009) 39:2076-82. doi:10.1002/eji.200939722

83. Imai T, Hieshima K, Haskell C, Baba M, Nagira M, Nishimura M, et al. Identification and molecular characterization of fractalkine receptor CX3CR1, which mediates both leukocyte migration and adhesion. Cell (1997) 91:521-30. doi:10.1016/S0092-8674(00)80438-9

84. Nishimura M, Umehara H, Nakayama T, Yoneda O, Hieshima K, Kakizaki M, et al. Dual functions of fractalkine/CX3C ligand 1 in trafficking of perforin+/granzyme B+ cytotoxic effector lymphocytes that are defined by CX3CR1 expression. J Immunol (2002) 168:6173-80. doi:10.4049/ jimmunol.168.12.6173

85. Teijaro JR, Turner D, Pham Q, Wherry EJ, Lefrancois L, Farber DL. Cutting edge: tissue-retentive lung memory CD4 T cells mediate optimal protection to respiratory virus infection. J Immunol (2011) 187:5510-4. doi:10.4049/ jimmunol.1102243 
86. Iijima N, Iwasaki A. T cell memory. A local macrophage chemokine network sustains protective tissue-resident memory CD4 T cells. Science (2014) 346:93-8. doi:10.1126/science. 1257530

87. Bottcher JP, Beyer M, Meissner F, Abdullah Z, Sander J, Hochst B, et al. Functional classification of memory CD8(+) T cells by CX3CR1 expression. Nat Commun (2015) 6:8306. doi:10.1038/ncomms9306

88. LaFleur C, Granados J, Vargas-Alarcon G, Ruiz-Morales J, Villarreal-Garza C, Higuera L, et al. HLA-DR antigen frequencies in Mexican patients with dengue virus infection: HLA-DR4 as a possible genetic resistance factor for dengue hemorrhagic fever. Hum Immunol (2002) 63:1039-44. doi:10.1016/ S0198-8859(02)00682-1

89. Sierra B, Alegre R, Perez AB, Garcia G, Sturn-Ramirez K, Obasanjo O, et al. HLA-A, -B, -C, and -DRB1 allele frequencies in Cuban individuals with antecedents of dengue 2 disease: advantages of the Cuban population for HLA studies of dengue virus infection. Hum Immunol (2007) 68:531-40. doi:10.1016/j.humimm.2007.03.001

90. Malavige GN, Rostron T, Rohanachandra LT, Jayaratne SD, Fernando N, De Silva AD, et al. HLA class I and class II associations in dengue viral infections in a Sri Lankan population. PLoS One (2011) 6:e20581. doi:10.1371/ journal.pone.0020581

91. Jessie K, Fong MY, Devi S, Lam SK, Wong KT. Localization of dengue virus in naturally infected human tissues, by immunohistochemistry and in situ hybridization. J Infect Dis (2004) 189:1411-8. doi:10.1086/383043

92. Durbin AP, Vargas MJ, Wanionek K, Hammond SN, Gordon A, Rocha C, et al. Phenotyping of peripheral blood mononuclear cells during acute dengue illness demonstrates infection and increased activation of monocytes in severe cases compared to classic dengue fever. Virology (2008) 376:429-35. doi:10.1016/j.virol.2008.03.028

93. Rothman AL. Immunity to dengue virus: a tale of original antigenic sin and tropical cytokine storms. Nat Rev Immunol (2011) 11:532-43. doi:10.1038/ nri3014

94. Fesnak $\mathrm{AD}$, June $\mathrm{CH}$, Levine BL. Engineered $\mathrm{T}$ cells: the promise and challenges of cancer immunotherapy. Nat Rev Cancer (2016) 16:566-81. doi:10.1038/nrc.2016.97

95. Buck MD, O’Sullivan D, Pearce EL. T cell metabolism drives immunity. J Exp Med (2015) 212:1345-60. doi:10.1084/jem.20151159

96. Cham CM, Driessens G, O’Keefe JP, Gajewski TF. Glucose deprivation inhibits multiple key gene expression events and effector functions in CD8+ T cells. Eur J Immunol (2008) 38:2438-50. doi:10.1002/eji.200838289

Conflict of Interest Statement: The authors declare that the research was conducted in the absence of any commercial or financial relationships that could be construed as a potential conflict of interest.

Copyright ( $(2016$ Tian, Sette and Weiskopf. This is an open-access article distributed under the terms of the Creative Commons Attribution License (CC BY). The use, distribution or reproduction in other forums is permitted, provided the original author(s) or licensor are credited and that the original publication in this journal is cited, in accordance with accepted academic practice. No use, distribution or reproduction is permitted which does not comply with these terms. 